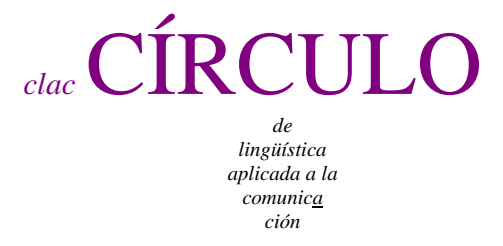

$55 / 2013$

\title{
PARENTÉTICOS, HEDGING Y SINTAXIS DEL ENUNCIADO
}

\author{
Catalina Fuentes Rodríguez \\ Universidad de Sevilla \\ cfuentes en us es
}

Resumen

El presente estudio constituye una revisión de algunas propuestas teóricas que intentan explicar la periferia del enunciado. Comment clauses, parentheticals y hedging son términos que categorizan aspectos distintos de la inscripción del hablante en el discurso y no todos tienen el mismo nivel de rentabilidad en un modelo global de gramática discursiva. Sin embargo, suponen un primer paso para exigir la descripción de una sintaxis del enunciado, que no esté cautiva de la todopoderosa sintaxis oracional.

Palabras clave: Sintaxis del enunciado, parentéticos, hedging, comment clauses.

Catalina Fuentes Rodríguez. 2013.

Parentéticos, hedging y sintaxis del enunciado. Círculo de Lingüística Aplicada a la Comunicación 55, 61-94.

http://www.ucm.es/info/circulo/no55/fuentes.pdf

http://revistas.ucm.es/index.php/CLAC

DOI: http://dx.doi.org/10.5209/rev_CLAC.2013.v55.43266

(C)2013 Catalina Fuentes Rodríguez

Círculo de Lingüística Aplicada a la Comunicación (clac)

Universidad Complutense de Madrid. ISSN 1576-4737. http://www.ucm.es/info/circulo 


\begin{abstract}
This paper reviews some theoretical proposals about sentence periphery. Comment clauses, parentheticals and hedging are terms which categorize different aspects of the speaker's assessment in the discourse and they have different levels of relevance in a global model of discourse grammar. However, they constitute a first step in order to claim the description of the sentence syntax, independent of the powerful clause syntax.
\end{abstract}

Key words: sentence syntax, parentheticals, hedging, comment clauses

Índice

2. Gramática y pragmática: el discurso 64

3. Comment clauses, ¿̇oraciones de comentario? 66

4. Hedging: subjetividad y aproximación enunciativa 74

5. Parentéticos: entonación, marginalidad y discurso referido 77

6. Las marcas del hablante y su visualización sintáctica en el enunciado 85

7. Conclusión 86

Bibliografía 87 


\section{Introducción}

La descripción de la sintaxis del enunciado ha ocupado a los especialistas en los últimos años, aunque en direcciones diferentes. La apertura de los estudios lingüísticos a la metodología pragmática, la atención a la sintaxis de lo oral y los trabajos sobre corpus han abierto una realidad muy compleja para la que los conceptos tradicionales de relación lingüística parecen insuficientes. El interés comenzó con la descripción de los “marcadores del discurso" por ser unidades aislables, poco descritas, y, por tanto, abarcables. Se podían describir sus características distribucionales, su semántica, su morfología, y su categorización, aunque en este campo no ha habido mucho consenso. Prueba de ello es la obra de Loureda-Acín (eds., 2010), en que se reúnen todas estas perspectivas. Pero no podemos olvidar que estas unidades solo cubren una parcela de lo que encontramos en el discurso, fuera de la estructura de la oración. El interés por seguir hablando de marcadores del discurso, a pesar de ser una etiqueta que vuelve a crear un cajón de sastre, viene de que en ella pueden incluirse, además de los conectores (relacionantes entre enunciados), otras unidades que sirven para expresar las coordenadas referentes al hablante y al oyente. Son lo que llamamos en su día operadores (Fuentes Rodríguez 2003). Pero en ese hueco sintáctico pueden aparecer muchas otras estructuras, consideradas periféricas (Fuentes Rodríguez 2005, 2007). Muchas de ellas sirven para expresar contenidos relativos a la inscripción del hablante dentro de su propio enunciado ${ }^{1}$.

En los últimos años han surgido varias propuestas para explicar dicha inscripción. Nos referimos a los estudios sobre comment clauses, hedging, o parentheticals. Tienen en común el ser un intento de explicación sintáctica de formas, unas más lexicalizadas, otras más libres, que aparecen como marginales en el discurso oral o escrito. En ocasiones la descripción se mueve en el plano de la sintaxis, en otras en la pragmalingüística, determinando los usos discursivos o valores modales. Pero no podemos obviar que se trata de propuestas que se acercan al enunciado, entendido este

\footnotetext{
1 Este trabajo se ha realizado dentro del marco del Proyecto FFI 2009-10515, del Ministerio de Ciencia y Tecnología y del Proyecto de Excelencia de la Junta de Andalucía P10-HUM 5872, ambos cofinanciados por fondos FEDER.
} 
como expresión mínima de una comunicación completa, e intentan describir su estructura. Nuestro interés aquí es exponer estos intentos y evaluar su rentabilidad para la elaboración de una sintaxis del discurso.

2. Gramática y pragmática: el discurso

La aparición de los enfoques pragmáticos, los estudios sobre actos de habla, cortesía, etc., han hecho frecuente la referencia a "funciones pragmáticas", "usos discursivos”, que apuntan no ya a contenidos denotativos sino a otros procedimentales donde el referente ya no es la predicación ni los argumentos verbales, con una designación en la realidad, sino contenidos relacionados con el hablante y el contexto. A este campo pertenecen los estudios sobre atenuación, formulación, discurso referido o expresión de la subjetividad.

La alternativa metodológica opuesta parece ser la gramática pura. Esta, en su versión más tradicional, no tiene “etiquetas” suficientes para denominar la formalización lingüística de estas coordenadas (Fuentes Rodríguez 1994, 2000, 2013), excepto cuando se verbaliza en morfemas (subjuntivo, diminutivo), o en un tipo de palabra como los adverbios (adverbios de modalidad, adverbios de enunciación...) Pero ¿y más allá de la palabra? Por ejemplo, ¿qué función desempeña “para serte sincero”, en el enunciado "Para serte sincero, aún no tengo trabajo fijo". ¿Es un complemento de finalidad? Una oración final de la enunciación (Galán 1999, RAE 2009, 3472-3475) sería la respuesta correcta, pero al decir esto, ¿estamos haciendo gramática, sintaxis discursiva, o pragmática? Aquí radica el problema. Para muchos investigadores más allá de la oración no hay sintaxis. Hay pragmática. Al decir esto, estos autores dejan a la pragmática abandonada en un limbo de "usos discursivos" no regulados, un espacio difuso que se extiende o se contrae según los intereses del investigador. Así, describiría pragmáticamente "para serte sincero” como una marca de la enunciación, pero tras esto no se lo suele analizar gramaticalmente. No importa que coincida en su estructura (para + infinitivo) con otras construcciones que tienen función final. La mayoría de estudiosos 
no se detiene en el análisis formal para llegar a una sistematización. Lo dejan todo a la “dependencia del contexto”. Consideran opuesto formalización y pragmática. Y si no hubiera codificación en la pragmática, no podríamos hablar de ello y la comunicación entre hablante y oyente no sería posible.

Hablar de un complemento de verbo enunciativo (Gutiérrez Ordóñez 1997, 2013) no siempre es admitido, porque supone considerar que hay una estructura de dependencias más allá de la oración. Y supone también resolver la espinosa pregunta de cuál es el tipo de relación que existe entre este “complemento” y su nudo, el verbo de decir implícito en todo enunciado (en tanto acto de habla realizado) ${ }^{2}$. En nuestra opinión, esta cuestión no puede ser obviada, puesto que existen estructuras perfectamente codificadas para expresar este contenido y esta función. El hueco existe, queramos verlo o no. Tiene formalización sintáctica, recurre a las mismas estructuras y construcciones que un complemento verbal cualquiera. Antes o después, tenemos que aceptar estos huecos sintácticos y ponerles nombre. Es decir, tenemos que extender la gramática al discurso y no pensar que la gramática tiene como marco la oración y la pragmática el discurso. ¿Tendríamos una disciplina del uso (i!) y otras (fonética, semántica, sintaxis) de la codificación? ¿O bien sería la pragmática la otra cara de la semántica? Se confunde, así, el uso con la necesidad de explicar estructuras mayores a la oración. Para las menores aceptamos la gramática como suficiente y no necesitamos, al parecer, explicar su realización discursiva. Solo recurrimos a esta (el discurso) cuando no tenemos etiquetas suficientes para estas secuencias mayores ${ }^{3}$.

Ante esta situación de falta de coherencia explicativa, muchos especialistas han elevado propuestas en torno siempre a estas formas que expresan la subjetividad del

\footnotetext{
${ }^{2}$ Rodríguez Ramalle $(2011,203)$, sin embargo, lo sitúa en el “sintagma fuerza” del margen izquierdo.

${ }^{3}$ El problema de fondo es la situación de la pragmática en relación con la Lingüística. Los que defienden esta posición consideran la pragmática una parcela que se añade a la gramática. Para otros, entre los que nos contamos, la pragmática es una perspectiva desde la que hacer lingüística, que contempla el uso junto al código, en todas las parcelas lingüísticas. Hay, pues, pragmática de lo fónico, de la sintaxis y de la semántica. Y pragmática no se opone a gramática, sino a gramática formal, ya que hay una gramática discursiva, contextual o pragmagramática, que es la que defendemos. Pero incluso desde el plano formal, hay que extender el campo de análisis a las unidades superiores a la oración.
} 
hablante, sus intervenciones metadiscursivas, a través de construcciones de diverso grado de fijación. Entre ellos los intentos más relevantes desde el punto de vista de su extensión internacional han sido los estudios sobre comment clauses, hedging y parentéticos, aunque estos no han llegado a ser considerados ni aplicados al español. Son propuestas con implicaciones distintas para la sintaxis, por lo que las revisaremos detenidamente, evaluando sus posibilidades explicativas y las conexiones que presentan con otros modelos. Nuestro esquema de trabajo será el siguiente:

- $\quad$ Comment clauses, ¿oraciones de comentario?

- $\quad$ Hedging: subjetividad y aproximación enunciativa

- $\quad$ Parentéticos: entonación, marginalidad y discurso referido

- $\quad$ Las marcas del hablante y su visualización sintáctica en el enunciado

- Conclusiones.

3. Comment clauses, ¿̇oraciones de comentario?

3.1. Quirk et al. (1980[1972]: 778-780; 1985: 1112-1120) definieron las comment clauses como: "parenthetical disjuncts that have a clausal structure and comment on the clause to which they are attached”. Este concepto supone una novedad, al menos para la gramática del español, que nunca ha considerado la relación de comentario dentro de las funciones que cumplen las cláusulas, y que cuando trata otras formas relativas a la subjetividad, se queda en el concepto de modalidad, y la circunscribe a los disjuntos (marcadores adverbiales), o bien a estructuras valorativas (modalidad explícita de Bally 1965). Por tanto, estructuras como lo que es más sorprendente, como dices, tú sabes, me imagino... no son etiquetadas bajo un solo nudo.

La pregunta que podemos hacernos es si la propuesta de estos autores se refiere a un tipo de relación libre entre oraciones, o bien a una estructura fosilizada, más cercana a los disjuntos, como parece deducirse de su definición.

Brinton (2008) ha relanzado el interés por las comment clauses analizando formas (I think, what's more, what's else...) que van gramaticalizándose para actuar como operadores de modalidad y de enunciación ( $I$ think), pero también en el plano 
argumentativo, como what's else, que invierte las expectativas creadas por la argumentación expresada por la secuencia que antecede.

Un paso más allá da Schneider (2007) cuando analiza en diferentes lenguas las RPC (reduced parenthetical clauses), estructuras más breves, que apuntan al decir (digamos, disons, je veux dire, diciamo...) y a la aserción (je crois, poni, volevo dire...). Él las analiza en español, italiano y francés. Ver que diferentes lenguas coinciden en la selección de estas marcas es interesante, ya que nos demuestra que estas actúan como una base de datos de la que el hablante toma formas para nutrir su paradigma de operadores de modalidad y enunciación. Habrá que analizar qué formas se gramaticalizan y elaborar un paradigma exhaustivo de estas unidades, marcando los diferentes grados del proceso en que se encuentran, así como sus diferentes campos de referencia.

El interés, pues, está en identificar formas que apuntan a la subjetividad, que cumplen la función de mostrar la implicación del hablante en lo dicho, y lo hacen a través de estructuras semifijadas, que vienen marcadas por la posición parentética.

Algunos otros, como Peltola (1982/83), han incluido aquí muchas más formas, como estructuras subordinantes condicionales, causales, etc... Su clasificación, que recoge Brinton (2008, 6-7), es la siguiente:

(a) inserted main clause, e.g. - there's no harm in naming him -

(b) sentence apposition, e.g., - worse luck!

(c) non-additive and clauses, e.g., and I know that they are great

(d) non-alternative or clauses, e.g., or so it seems

(e) non-conditional if clause, e.g., if I refrain from discussing these questions, if you'll forgive the expression

(f) parenthetic as clause, e.g., as I had thought, as far as I can tell

(g) parenthetic relative clause, e.g., what is more, which sometimes happens

(h) elliptical predicative in front position, e.g., no wonder, more important

(i) interjection, e.g., thank God, 'fortunately' 
(j) adverb, either opinionative, e.g., understandably ( $<$ it is understandable), signaling, e.g., honestly, or modal, e.g., possibly (cf. I think/suppose/imagine)

(k) prepositional phrase, e.g., in summary (cf. to sum up)

(l) parenthetic epistemic main clause, e.g., I guess, one wonders, it is asserted, he argues

(m) absolute use of the infinitive, either modal, e.g., truth to tell, opinionative, e.g., to give him his due, or signaling, e.g., to begin with

(n) absolute use of the present participle, e.g., judging by the headlines. Peltola (1982/1983: 103ff)

Se incluyen, pues, todos los segmentos que podemos encontrar en posición marginal (Fuentes Rodríguez 2005, 2007, 2012). Es decir, tanto los incisos relacionados con la oración matriz, como los segmentos intercalados en ella, que no tienen relación sintáctica con el verbo de la oración, es decir, los parentéticos (Fuentes Rodríguez 1998). Esta extensión nos lleva a dudar de la rentabilidad del concepto de comment clause así entendido. ¿Deberíamos, pues, reducirlo a las diferentes estructuras utilizadas para designar el decir y la subjetividad? ¿Qué incluiríamos bajo este concepto? ¿Estructuras semifijadas, como las RPC de Schneider (2007), es decir, construcciones oracionales que tienen esta función de marcar la modalidad y que están en vías de convertirse en fórmulas fijadas? De esta manera, su rentabilidad consistiría en ser el concepto bajo el que incluir estructuras como: me parece, imagino, creo, tú sabes, como te digo,... Pero también formas como lo que es mucho decir, lo que es más sorprendente... y otras que introducen una relación significativa de comentario: establecer un contenido valorativo sobre lo anterior. Son dos posiciones diferentes.

3.2. Optar por la primera opción, considerar comment clauses la denominación específica de las estructuras semifijadas que marcan modalidad y enunciación, nos llevaría a considerarlas el estadio intermedio en la gramaticalización de los marcadores discursivos. Un proceso que va desde las oraciones subordinadas que actúan en un plano marginal del enunciado (si me permites que añada un dato más, para no insistir más en ello, lo que es mucho más raro de explicar...) hasta los operadores discursivos (por favor, ¿sabes?). Por ejemplo, en el campo de la enunciación, podemos tener operadores como francamente, sinceramente..., completamente fijados en esta función, con un comportamiento claro: aparecen entre pausas, son marginales sintácticamente, no 
funcionan dentro de la oración, se refieren a la actividad del hablante en tanto tal...

(1) Sinceramente, ¿trató de cuidarse pese a la lesión? (CREA, El Mercurio, 16/07/2004)

(2) La verdad, desde que estoy en el Real Madrid, los entrenamientos de pachangada son bastante raros. (CREA, La Voz de Galicia, 15/01/2004) ${ }^{4}$

Y en un paso previo de gramaticalización se situarían las comment clauses, entre las que incluiríamos pienso, creo, me parece, sospecho..., insisto.

(3) Al final de mi estancia en Roma, empecé a estar muy preocupado, a deprimirme, porque el Cervantes funcionaba fatal -y sigue funcionando fatal, creo- y por el parón que se produce en mi producción intelectual y científica, por mi papel de burócrata, sumergido en temas administrativos. (CREA, Revista Telos. Cuadernos de Comunicación, Tecnología y Sociedad, 01-03/2004, n58, Segunda Época)

(4) El único argumento -me parece- para plantear la "opción" de un matrimonio homosexual encuentra asidero en cierta victimización de los homosexuales y en una manipulación del principio de no discriminación. (CREA, El Mercurio, 08/03/2004)

(5) En estos momentos, he de trasladarles que Andalucía lidera la gestión de los fondos Jeremie en la Unión Europea, con casi un 25\% del total de los fondos de esta iniciativa ejecutados hasta este momento, a nivel - insisto- de toda la Unión Europea. (DSPA ${ }^{5}$ 111, 19, sr. Ávila Cano)

Tengamos en cuenta que estas construcciones empiezan a desarrollar esta función de comentario en posición intercalada y final, entre pausas, y no suelen aparecer en posición inicial, frente a los adverbios anteriores, operadores $\mathrm{ya}^{6}$, que sí tienen movilidad posicional. Al inicio del enunciado suelen actuar generalmente como un segmento integrado. Sin embargo, hemos localizado en nuestro corpus de discurso

\footnotetext{
${ }^{4}$ Para este trabajo hemos utilizado el Corpus de Referencia del Español Actual, de la RAE (corpus CREA), así como las sesiones del Parlamento Andaluz correspondientes a la última legislatura (http://www.parlamentodeandalucia.es/webdinamica/portal-web-arlamento/recursosdeinformacion/ diariosdesesiones/comisiones.do).

${ }^{5}$ DSPA: Diario de Sesiones del Parlamento Andaluz.

${ }^{6}$ Cfr. Fuentes Rodríguez (2003). Los operadores son elementos, generalmente adverbios en su origen, cuya función no es modificar semántica ni funcionalmente a verbos, adjetivos o adverbios, sino aportar una indicación macroestructural sobre el acto enunciativo, la modalidad, la estructuración de la información o de la argumentación.
} 
parlamentario $^{7}$ un caso en el siguiente fragmento:

(6) Insisto, no los políticos, en general, ni el Partido Socialista, que también, sino este Gobierno. (DSPA 43, 47, sr. Ferreiras)

El parlamentario introduce una aserción que viene a reforzar lo dicho anteriormente.

Estas comment clauses no tienen todas el mismo grado de fijación. Así, podríamos considerar intuitivamente creo o me parece más evolucionados ${ }^{8}$ que sospecho, todavía una estructura libre, por lo que hablaríamos de parentéticos... Todas coinciden en la expresión de una gradación de la responsabilidad asertiva...Veámoslo con un ejemplo:

(7a) Dicen que Joaquín se ha comprado el piso de abajo

(7b) Joaquín, según me dicen/ por lo que he oído, se ha comprado el piso de abajo

(7c) Joaquín, sospecho, se ha comprado el piso de abajo

(7d) Joaquín, me dicen, se ha comprado el piso de abajo

(7e) Joaquín, creo, se ha comprado el piso de abajo

(7f) Al parecer, Joaquín se ha comprado el piso de abajo

El proceso de fijación sintáctica podría establecerse del siguiente modo: Frente a una construcción libre, un enunciado formado por dos oraciones en relación de interdependencia $(\mathrm{v}+\mathrm{cd})$ que expresa en el contenido léxico del verbo, la evaluación o el decir (7a), podemos tener otras formas de manifestación que caminan hacia lo más fijado que es el operador discursivo (7f). Las etapas de esta evolución implican diferentes construcciones sintácticas: complementos periféricos marginales (7b), enunciados parentéticos y discurso referido (7c y 7d) comment clauses en 7e y operador en $7 f$.

Constituyen una escala que va de la menor fijación a la mayor y que supone que en los estadios intermedios que van de la construcción oracional hasta el operador discursivo (los dos extremos están estudiados y admitidos por la gramática), hay otras construcciones sintácticas que explicar. En este estadio intermedio podría insertarse la

\footnotetext{
${ }^{7}$ Cfr. nota 4.

8 Evidentemente, esto exige un estudio detenido. Pero confróntese con Schneider (2007) y Fuentes Rodríguez (2010), donde se muestra su elevada presencia en el ámbito parlamentario.
} 
comment clause, pero también los periféricos (Fuentes Rodríguez 2007, 2012, Gutiérrez Ordóñez 1997, Dik 1997) o el discurso referido. Y los parentéticos, que, como vamos a ver más adelante, responden a dos enunciados en el que uno interrumpe al otro, en una relación de inserción, en la que el intercalado señala la modalidad o la responsabilidad asertiva ante lo dicho.

Comment clause podría ser, pues, un concepto aplicable a una estructura sintáctica marginal en un grado avanzado de gramaticalización camino de convertirse en operador modal, enunciativo o argumentativo.

3.3. Además de esta interpretación y como segunda opción, el concepto de comentario (oración de comentario) puede ser entendido como un contenido (semántico o gramatical) que tiene que ser considerado en nuestra gramática en la tipología de la relación interoracional. El comentario no es exactamente modalidad, entendida en el sentido clásico del término (Bally 1965, Zavadil 1968, Palmer 1986, Fuentes Rodríguez 1991), sino que designa una operación semántica en la que el hablante expresa su evaluación sobre el contenido precedente. No es una expresión de su emoción o su subjetividad, sino un juicio de valor. Podría suponer en parte la gramaticalización de contenidos relacionados con lo que hoy se llama “appraisal”: White (2000, 2002, 2003, 2004), Martin (1995, 2000, 2003, 2004), Martin-White (2005), y Hunston-Thompson (2000), Kaplan (2004) y González Rodríguez (2011) ${ }^{9}$. Incorporar este tipo de contenido relacional puede ser muy rentable dentro de la sintaxis discursiva, ya que cubre un ámbito designativo en el que operan formas como adverbios disjuntos, estructuras en proceso de gramaticalización y relaciones entre oraciones, marcadas por diferentes conectores: $y, o$, relativos... El segundo segmento establece una valoración sobre el contenido de la primera. En este sentido podríamos incluir adverbios como curiosamente, asombrosamente..., considerados “content disjuncts" que expresan “value judgement” (Quirk et al. 1985, 620 y ss....) y por Kovacci (1992, 159)

\footnotetext{
${ }^{9} \mathrm{El}$ tema de la evaluación es ahora un campo de análisis fructífero, donde abundan los acercamientos desde diversos puntos de vista. Consúltese, asimismo, Lemke (1992, 1998), Caffi-Janney (1994), Englebretson (ed., 2007), la perspectiva de Kärkkäinen (2003), o los estudios encuadrados en NiemeierDirven (eds. 1997)
} 
“adverbios omisibles valorativos” o sintagmas como el que resaltamos en el siguiente fragmento.

(8) Este, señor Oña, le pese lo que le pese, es el partido de la honestidad, que, en cuanto hay alguien que comete un delito, se va a la calle, y se ayuda a la Justicia, cosa que ustedes no hacen.(DSPA 111, 26, sr. Núñez Roldán)

Este contenido es fundamental para las oraciones y estructuras que aparecen en el margen derecho de la oración, como demostramos en Fuentes Rodríguez (2012), y como algunos expertos dejan entrever al hablar de afterthought, o cuando establecen los contenidos de los “extraclausals constituents” (ECCs). Dik (1997, 311), por ejemplo, considera que estos ECCs cubren las siguientes funciones pragmáticas: “(i) the 'management' of the interaction, (ii) 'comments' on the content of the clause proper, and (iii) the organization of the content of the expression, in relation to the context in which it occurs".

La relación de comentario está incluida en las relaciones semánticas que contraen las oraciones. Pertenecería a un proceso de expansión y elaboración. Así aparece en la propuesta de Halliday (1985: 202 y ss.), quien, desde la perspectiva funcional, separa la versión sintáctica de la relación como interdependencia, de la lexicosemántica, en la que se entiende la oración compuesta como un sistema basado en la expansión y proyección:

A) Expansión: la cláusula secundaria expande la primera,

aa) elaborándola (especifica detalles, parafrasea, comenta o ejemplifica),

ab) extendiéndola (añade nuevos elementos, da una alternativa o una excepción), 0

ac) realzándola, intensificándola o aumentándola (la califica con circunstancias de tiempo, causa, lugar o condición).

b) Proyección: la cláusula secundaria puede constituir la proyección

ba) de una locución o de una idea.

bb) de palabras: estilo directo, indirecto o libre. Entre ellos diferencia "quoting” (discurso directo), que es un proceso verbal, expresado por relaciones 
paratácticas, y “reporting” (discurso indirecto), proceso mental, expresado por relaciones hipotácticas.

La Segment Discourse Representation Theory (SDRT), por su parte, analiza las relaciones discursivas como relaciones retóricas: "the principles which account for textual cohesion. Such relations are, e.g., Narration, Parallel, Contrast and Explanation (Hobbs 1985, Mann \& Thompson 1986, Polanyi 1995, Kehler 2002, Asher \& Lascarides 2003” (Leth, p. 1).

Asher-Vieu (2005) parten de que el comentario es una relación subordinante, tal como es habitualmente clasificada:

“-Coordinating: Narration, Background, Result, Continuation, Parallel, Contrast. In dialogue, Question-Coordination and Correction have also typically been assumed to be coordinating.

-Subordinating: Elaboration, Instance, Topic, Explanation, Precondition, Commentary

For dialogue, we would include Question-Elaboration and (Indirect) QuestionAnswer Pair as well”. (Asher-Vieu 2005, 596)

Tras un análisis en el que somete el concepto a los tests correspondientes afirma: "Elaboration, Explanation and Commentary are apparently always subordinating, but they don't share any common semantic or information content properties that coordinating relations don't share. This confirms our hypothesis that relations aren't subordinating or coordinating in virtue of their content but rather in terms of how they are presented in the discourse” (606)

Por tanto, desde el punto de vista de la construcción textual, existe un tipo de conexión lógico-semántica (o cognitiva) entre las oraciones, entre las que se incluye el comentario. Sin embargo, no se tiene en cuenta a la hora de tipificar las relaciones entre oraciones. ¿Es que no tiene visualización sintáctica entre las oraciones coordinadas o subordinadas? O mejor, ¿̇no hemos tipificado aún en español la formalización de esta relación?

En mi propuesta de 2010, publicada en 2013 (Fuentes Rodríguez 2013), considero 
que la relación semántico-discursiva de comentario puede verse manifestada de diversos modos, que cubren desde el empleo de operadores evaluativos como paradójicamente, curiosamente (9a), hasta sintagmas y oraciones (9b y 9c):

(9a) Curiosamente, la solicitud se había perdido

(9b) Habían vuelto a considerar la solicitud de Antonio, algo muy sospechoso

(9c) Habían vuelto a considerar la solicitud de Antonio, lo cual no era en absoluto normal

En suma, el concepto de comentario debe ser incorporado como un tipo de conexión discursiva entre las oraciones. Pero necesitaríamos otro término en español para designar las comment clauses como estructura intermedia previa a la fijación del operador discursivo, ya sea modal, enunciativo o argumentativo. RPC podría ser una alternativa (o CPR, en español: Cláusula parentética reducida).

\section{Hedging: subjetividad y aproximación enunciativa}

Otra etiqueta sintáctica que surge en la bibliografía al analizar el comportamiento discursivo de marcas relacionadas con el hablante es el de hedging. Este concepto ha ido ganando adeptos dentro de la literatura anglosajona, desde la propuesta que hizo G. Lakoff en 1972. Incluso Santos Río (2003) utiliza el concepto en su traducción (“cerca semántica”) para describir algunas partículas. Sin embargo, es un término ambiguo, no solo por los elementos que abarca, sino también por el campo significativo y/o funcional que incluye. Los hedges son generalmente partículas, o estructuras cuasigramaticalizadas, elementos que tienen una función macroestructural y que se mueven en dos planos: el del significado vago (fuzziness) y el de la intervención, actitud o compromiso del hablante. Lo que tienen en común estos dos campos es precisamente la relación de lo dicho con la visión subjetiva del hablante. O, mejor dicho, con la calificación de la aserción.

Comienza entendiéndose como una marca atenuativa sobre el decir (Weinreich 1966), para reformularse en Lakoff (1972) como etiqueta para expresiones de habla 
borrosa o aproximativa: sort of, kind of, technically speaking, strictly speaking. Para él hedge es una palabra "whose job is to make things fuzzier or less fuzzy"10. Su efecto recae en las condiciones de felicidad de los enunciados y con la propia estructura conversacional. Pueden actuar sobre los performativos, generalmente con efecto mitigador. Fraser (1975) confirma esta posibilidad de actuar como modificador de marcas de expresión de la actitud del hablante.

De esta manera se perfila el doble valor que va a tomar este término en la bibliografía a partir de 1978. Prince et al (1982) ya distinguen entre fuzziness del contenido proposicional (“propositional hedging”: “estaba como demacrado”) o de la relación entre dicho contenido proposicional y el compromiso del hablante con la verdad de la proposición (“speech act hedging”: “debo advertirte que...”, creo que...). En esta propuesta, y en la de Brown-Levinson (1987[1978]) el "speech act hedging” puede usarse con un fin atenuativo o reforzador. Su definición es: “A hedge is a particle, word or phrase that modifies the degree of membership of a predicate or noun phrase in a set; it says of that membership that it is partial, or true only in certain respect; or that it is more true and complete than perhaps might be expected” (Brown-Levinson 1987, 145). Su función atenuadora lo acerca directamente a la cortesía. En este sentido se entiende en el trabajo de Briz-Estellés (2010).

Esta relación hace que el concepto se mueva entre lo gramatical, como una etiqueta discursiva para designar a cierto paradigma de formas que no estaban bien integradas en la gramática (los aproximativos, Fuentes Rodríguez 2008), o como un elemento que quiere emplearse como archilexema para todo lo relacionado con la mitigación o la intensificación, un contenido discursivo.

Así parece derivarse de la propuesta de Fraser (2010, 15), que considera el hedging una estrategia retórica, ligada a la competencia pragmática, "that attenuates either the full semantic value of a particular expression, as in He's sort of nice, or the full force of a speech act, as in I must ask you t stop dong that”. Sería, pues, una nueva etiqueta relacionada con la estrategia de mitigación (Caffi 1999, 2007, Meyer-Herman

\footnotetext{
${ }^{10}$ Por ejemplo una especie de lo hace borroso, pero técnicamente hablando le da fuerza.
} 
1988, Briz 1998, Schneider 2013,...). De esta manera puede usarse para denominar muchas estructuras y elementos de alcance y categoría diferentes: desde diminutivos, verbos modales, hasta operadores o construcciones más extensas (creo que, me parece, estrictamente hablando, por decirlo así...). No podríamos, pues, usar hedges como etiqueta denominativa de una clase funcional de unidades que tienen un comportamiento discursivo determinado, ni de una estructura o relación funcional.

Es un término, pues, utilizado para designar la intervención del hablante en su discurso mostrando la adecuación o inadecuación de lo dicho al interés del que habla. Atiende a la formulación que hace este y a sus efectos discursivos. Usar el término hedge intenta darle unidad a todos estos valores, que de este modo parecen conseguir categorización sintáctica. Sin embargo, hay gran diferencia entre lo que es aproximación formulativa (una especie de...), y la atenuación marcada por expresiones modales (creo, imagino...) Tampoco hay unidad categorial, porque el hedging puede ser entendido como un fenómeno pragmático que se expresa a través de formas fijadas, adverbios, y operadores, o construcciones libres.

Por tanto, hablar de hedging es hablar de mitigación o atenuación. Pero no nos resuelve mucho en sintaxis discursiva. No nos proporciona etiquetas formales para clasificar, determinar una clase funcional de unidades, o un hueco funcional. Termina, pues, siendo un término muy genérico, que no aporta mucho al concepto de atenuación, y que deja fuera muchas otras formas relacionadas con la expresión de la subjetividad del hablante. Pero constituye otro testimonio del intento de los estudiosos de encontrar una etiqueta para designar las construcciones y elementos sintácticos que visualizan la inscripción del hablante y su subjetividad en el discurso (cómo enfoca la realidad, qué estilo de habla, de designación, de exposición, elige...), y, al mismo tiempo, evidenciar que este comportamiento sintáctico es específico y exige etiquetas nuevas.

En mi opinión, hedging es el resultado de unir en un solo concepto aspectos que surgen de la macroestructura discursiva y buscar un término que en sintaxis aborde toda esta diversidad. Quizás sería más rentable utilizar el concepto de operador para lo específicamente sintáctico y matizar luego su campo de acción. Por otra parte, si lo que se pretende es atender al significado borroso o vago, no debe mezclarse con otros 
elementos como I think, you know, cuya función discursiva es diferente. Sin embargo, sí hay que reconocerle a Lakoff que incorporar las formas fuzzy, como un estilo de habla, hace que tengamos que plantearnos si la atenuación es una estrategia modal o formulativa. Sería mejor considerarla argumentativo-formulativa, un punto donde enunciación y argumentación confluyen, como todo en el discurso. El hablante no tiene parcelas, habla como un ser total, integral, con todas sus emociones e intenciones de forma simultánea. El análisis debe ser, por tanto, multidimensional.

5. Parentéticos: entonación, marginalidad y discurso referido

Otro intento de encontrar instrumentos sintácticos para la explicación de los niveles supraoracionales es el interés por los parentéticos. Esta es una propuesta más limitada, y más centrada en la formalización. Pero la base de su utilización sigue siendo la misma. En Dehé-Kavalová (eds., 2007) se parte de la siguiente descripción:

«Parentheticals are expressions that are linearly represented in a given string of utterance (a host sentence), but seem structurally independent at the same time. They have been argued to interrupt the prosodic flow of an utterance, introducing intonational breaks and featuring prosodic properties different from those of their host. They are outside the focus-background structure of their host utterance and are usually associated whith non-truth conditional meaning. Parentheticals typically function as modifiers, additions to or comments on the current talk. They often convey the attitude of the speaker towards the content of the utterance, and/or the degree of speaker endorsement» (Dehé-Kavalova, 2007: 1).

Se trata, por tanto, de estructuras relacionadas con la presencia del hablante en el discurso.

En 2012 se organiza un Coloquio en París con este nombre, y reúne a especialistas 
de diversas partes del mundo, que tienen en común el ocuparse de "comment clauses", marcadores discursivos y discurso referido, fundamentalmente. En el fondo, de formas relacionadas con el decir y la subjetividad, que, además, no tienen una caracterización sintáctica “estándar”: no pueden ser explicadas en relación con el verbo de la oración. Sin embargo, aquí la realidad es quizás mucho más compleja y abarcadora que en los casos anteriores y está centrada en lo sintáctico.

Una propuesta paralela es la de Blanche-Benveniste (2002, 2003), que analiza los elementos marginales del enunciado como préfixes, infixes y suffixes, según su posición. Kahane-Pietrandrea (2011) habla de unités illocutoires associées. En estas prima más el deseo de buscar la explicación sintáctica al comportamiento de estos segmentos y estructuras $^{11}$.

Generalmente, el concepto de parentético ha sido utilizado en la gramática española pocas veces. Aparece como marca distribucional y/o entonativa de los marcadores del discurso, y de ciertas funciones marginales dentro del enunciado. Los teóricos de la función incidental (Martínez 1994 y Fernández 1993) utilizaron la pausa como marca sintáctica de complementos periféricos. Abarcó adjetivos (los incidentales de Lapesa, 1975), adverbios (atributos oracionales de Alarcos 1973) y oraciones “subordinadas impropias” (los modificadores de modalidad de Kovacci 1992 o los circunstantes de Gutiérrez Ordóñez 1997). Sin embargo, en otras tradiciones lingüísticas el estudio de los parentheticals se ha dirigido hacia las marcas de modalidad (DehéKavalová 2007, Brinton 2008, Kaltenbock 2007, ed. 2010, vid. Schneider 2007, 20 y ss.) o el discurso directo (Schelfhout 2000, Wichmann 2001).

Como bien dice Espinal, "considered in isolation, there is nothing particular which identifies a parenthetical” (Espinal 1991: 728). Para Kaltenböck (2007) surge de su relación con la oración host. Este autor intenta realizar una caracterización de las

\footnotetext{
${ }^{11}$ Últimamente se han publicado estudios sobre el margen izquierdo (Rodríguez Ramalle 2011) o López Serena (2012) sobre la dislocación. Ambas son propuestas parciales, pero que muestran el interés por este ámbito sintáctico.
} 
diferentes estructuras sintácticas que pueden aparecer como "parenthetical clauses”, definidas por su falta de relación sintáctica con la oración base, su estructura clausal, y su movilidad posicional. Esto le lleva a delimitar los siguientes grupos situados en tres ámbitos, correspondientes a los tres círculos concéntricos de la siguiente figura. El círculo más exterior es de la "clausal form”, el intermedio el de "syntactic independence”, y el más interior la “positional flexibility” (Kaltenböck 2007, 49):

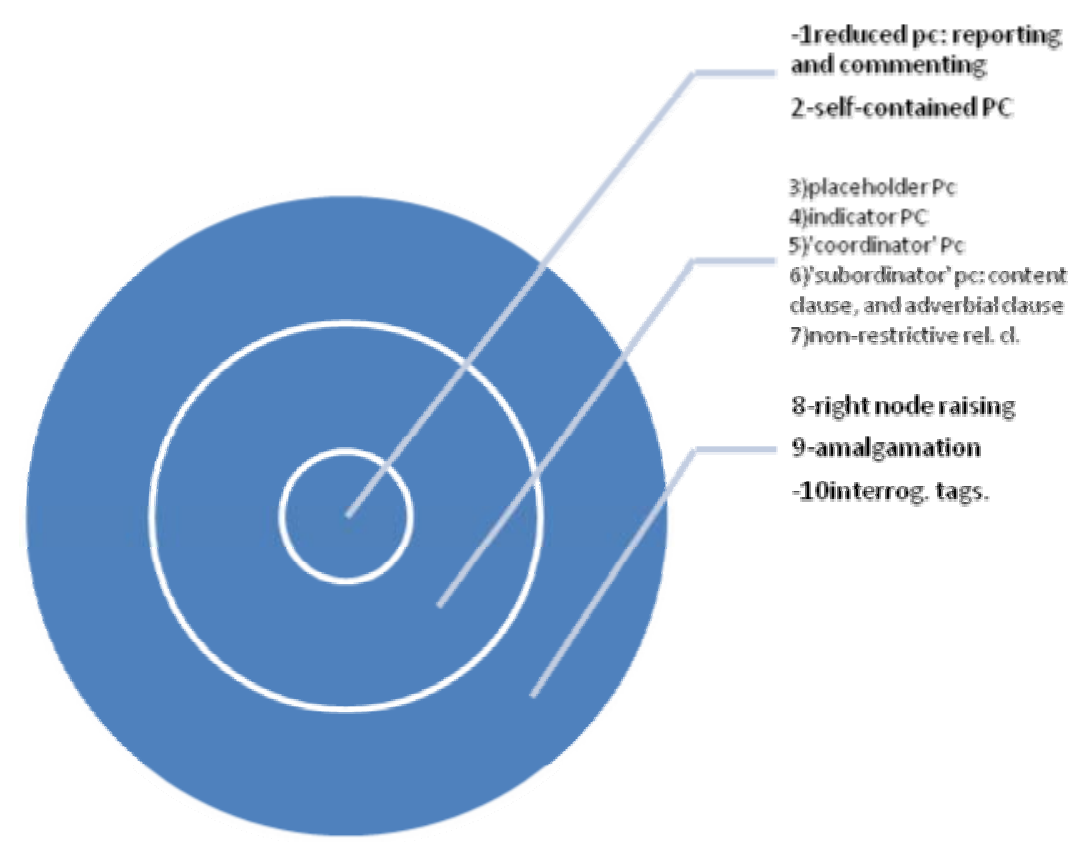

1- Britons he said could compete and win (ICE-GB: s2b-005, \#129)- (Kaltenböck, p. 41)

2- Mary- I hate to tell you this- is coming over to visit (p. 40)

3- You're your <,> uh what's this called thorax <,> is probably vibrating far too much (ICE-GB: s1a-018 \#53). (p. 41)

4- Many clauses are syndetic, that is they do not have an overt marker (p. 38)

5- For several years now -and I don't mean to be cynical- we have been trying without success to overcome these problems (p. 39)

6- The warning -that prices should be lowered- was ignored (p.39) 
7- Mary is away on business, which is convenient. (p.39)

8- He is, or at least he was, a great actor (p. 30)

9- He gave this I prefer not to know how awful paper (p. 30)

10- Mary is coming tomorrow, isn't she? (Kaltenböck 2007, 30)

Para Kaltenbock (2007) la relación entre la cláusula parentética y su oración base no es de dependencia ni de integración. Es un adyacente, que "intersect with the host structure purely on the linear axis and are not linked by any syntactic nodes - a fact that has proved to be a particular problem for models of syntactic representation” (Kaltenböck 2007, 35). Nosotros hablamos en 1998 de interrupción de un enunciado sobre otro, motivado por la necesidad de expresar características de procesamiento. Su función, pues, es de contextualización y anclaje del contenido proposicional en las coordenadas enunciativas. Por supuesto supera la estructura oracional, y apunta a la necesidad de plantear otra estructura superior: la del enunciado. Nuestra caracterización se centró en los enunciados parentéticos, que aparecen determinados por la semicadencia, frente a los incisos, que terminan en semianticadencia (Fuentes Rodríguez 1998), ya que responden a complementos de la oración matriz.

El enunciado parentético muestra un comentario o unas marcas relativas al hablante, a su subjetividad o a las instrucciones de procesamiento de la información: qué elementos son más destacados o cuál es su función argumentativa.

Esta categorización es, pues, más estricta y se reserva el nombre de parentético para un tipo de relación de no inserción que se muestra formalmente por la presencia de la pausa. Constituiría uno de los tipos de construcción periférica.

Veamos unos ejemplos elegidos al azar de un diario de sesiones del Parlamento Andaluz:

(10) Con respecto a los proyectos de las corporaciones locales, señor Fuentes, su lugar es la Ley de Ordenación del territorio, donde se incluyen como proyectos estratégicos - y se lo cito textualmente«las actuaciones de carácter público consideradas de especial relevancia como consecuencia de su magnitud, su proyección económica o social, o su importancia para la estructuración territorial de Andalucía». (DSPA 119, 12, sr. Núñez Roldán) 
"Y se lo cito textualmente" es un enunciado parentético que interrumpe al anterior y muestra las circunstancias metadiscursivas de su emisión. Va unido con y al enunciado previo ¿cuál es el lugar de inserción de esta coordinación? ¿dos actos (MackenzieGómez González 2004)? ¿Dos oraciones complemento de un implícito digo? Su relación estaría en el nivel más alto, en el plano del decir: “digo X y lo digo textualmente”. Desde nuestro punto de vista serían dos enunciados.

En el siguiente el hablante inserta una relativa seguida de un enunciado de comentario. Introduce un argumento más una valoración del hablante.

(11)Yo no sé qué razón habrá para que el señor Fuentes Lopera — que dice que la ley no es mala; yo creo que es lo más amable que ha dicho en esta cámara- esté en contra de votar a favor de una ley que se llama «proyecto para potenciar inversiones empresariales de interés estratégico para Andalucía y de simplificación, agilización administrativa y mejora de la regulación de actividades económicas en la comunidad Autónoma de Andalucía» (DSPA 119, 12, sr. Núñez Roldán).

Un caso más claro de explicar desde el punto de vista sintáctico es cuando lo insertado en el paréntesis es un añadido informativo relevante, expresado en forma de sintagma circunstancial. La marginalidad viene expresada por la curva melódica propia del paréntesis y su semicadencia, lo que muestra que es un añadido de algo no planeado previamente. En el mismo caso están otras estructuras que sintácticamente toman la visualización de sintagmas u oraciones dependientes.

(12)Y por eso, por última vez, les solicito — después del esfuerzo, yo creo, de generosidad que el Grupo Socialista ha hecho aprobando el 92,3\% de las enmiendas de Izquierda Unida y cinco de las 13 enmiendas del Grupo Popular - que ustedes reconsideren su actitud, que aprueben, que lancemos un mensaje de confianza y de esperanza al tejido productivo andaluz y a todas las familias andaluzas, cuya mayor preocupación es que se cree empleo. (DSPA 119, 12, sr. Núñez Roldán)

(13) Y una segunda reflexión sobre la soberanía: este decreto ley es de adaptación a Andalucía de una legislación estatal que no ha sido demandada por la ciudadanía, que no aparece en ningún programa electoral —especialmente, del partido que está gobernando- y que se ha puesto en marcha para satisfacer la voracidad de los llamados mercados - que estamos deseando que nos los presenten- y para satisfacer los deseos de inversores privados de fondos de reptiles y de la gran banca de este país. Al frente de la gran banca de este país creemos que lo que hay son delincuentes que deberían —si estuviésemos en una democracia de verdad_..., deberían estar siendo ahora mismo 
juzgados por la ciudadanía, por lo que han hecho y lo que siguen haciendo impunemente. (DSPA 119, 18, sr. Mariscal)

Todos estos segmentos no pueden entenderse como complementos del verbo de la oración matriz, ya que la disposición entonativa del paréntesis muestra la intencionalidad del hablante de presentarlo como información de segundo plano, fruto de la intervención de otro enunciador (desdoblamiento del hablante) y como comentario de lo previo. Son dos enunciados que se interrumpen para añadir, comentar o corregir. En otra ocasión el parentético alberga marcas cohesivas o de procesamiento: y voy terminando, que actúan como elementos de engarce, de cohesión en el discurso.

(14) Otras modificaciones introducidas $-y$ voy terminando-, que pretenden reforzar el gobierno corporativo de las cajas, hacen referencia a la prohibición de que los miembros de gobierno de los órganos puedan vincularse a la caja por alguna relación retribuida en los dos años siguientes a su cese, lo cual se extiende a las fundaciones de la caja y sociedades en las que se participe directa o indirectamente. (DSPA 119, 17, sr. Ávila Cano)

Comparemos esta estructura, que termina en semicadencia con estas otras construcciones que aparecen en inciso, como complementos periféricos, pero no constituyen enunciados parentéticos:

(15) Sabemos que está ya en su casa, y desde aquí, que nos estará viendo, porque es muy nervioso, le deseamos, como digo, en nombre del Grupo Parlamentario Popular y de mi portavoz y Presidente, Javier Arenas, un pronto restablecimiento (DSPA 117, 8, sr. Fuentes Lopera)

Muchas de ellas, como hemos visto, son estructuras originariamente subordinadas, que actúan como marcas de procesamiento. La mayoría introduce argumentos, restricciones informativas, comentarios, o circunstancias del decir. Incluso de cohesión del contenido del discurso. De nuevo, marcas que en un primer momento saltan desde el interior de la oración (oración subordinada) al interior del enunciado (complemento periférico) y de ahí pasan a actuar como conector entre enunciados.

Tener en cuenta la relación parentética nos abre un panorama más amplio, porque vemos la posibilidad de insertar no solo marcas de procesamiento, sino también otras informaciones en una doble enunciación, fruto del desdoblamiento del hablante (Fuentes Rodríguez 1998) en dos enunciadores. Un ejemplo claro es el siguiente: 
(16) Pero hay más, señor Consejero, ¿sabía usted — claro que lo sabe-que la presidenta del comité de empresa de Intersur, mientras que se gestionó ese ERE, con los intrusos y con los infiltrados de Camas, ahora mismo es concejal del Partido Socialista en Morón? ¿Sabía usted —claro que lo sabía - que la premiaron ustedes poniéndola la número tres en la lista, y que hoy es concejala del Partido Socialista en Morón, señor Consejero? ¿Eso es colaborar con la justicia? (DSPA 112, 27, sr. Raynaud)

Nos permite plantearnos cuál es la relación sintáctica entre los dos enunciados, al mismo tiempo que presentar lo dicho en un doble plano: hay una jerarquía entre las dos informaciones. ¿Pero lo podemos explicar como primer plano y fondo? Creo que el hablante quiere presentarlos en la misma jerarquía de presencialidad aunque con funciones distintas. La estructura sería: Información base + información insertada (la del parentético), que corresponde a las instrucciones de procesamiento o a los añadidos argumentativos necesarios para llevar al oyente a la conclusión que quiere el hablante, corrigiendo, invirtiendo o intensificando lo que el enunciado base propone.

¿A qué apunta todo lo visto? A la necesidad de ampliar la sintaxis si queremos explicar el discurso. Este no es una simple suma de oraciones (sería así si consideramos que la unidad base y techo es la oración) ${ }^{12}$. La unidad base, de entrada, es el enunciado realizado. Necesitamos, pues, utilizar criterios pragmáticos, porque nos estamos moviendo en la realización. ¿Y por qué? Porque si seguimos obviando esto nunca describiremos cómo se formalizan las marcas de contextualización. Algunas son tan internas al elemento lingüístico como los morfemas verbales o los pronombres. Hay otras que son más “externas”, más "libres o independientes entonativamente o funcionalmente”, pero no por ello deben dejar de describirse.

Ampliemos, pues, la mirada, y afrontemos la existencia de una unidad, el enunciado, que incluye, junto a la oración (u oraciones) que expresan el contenido informado, otras marcas relativas al procesamiento de dicha información. Este apunta, por un lado, al hablante-oyente, y, por otra, al contexto externo e interno, es decir, al resto del discurso en el que se sitúa lo dicho. La formalización de esa parcela marginal, o de anclaje contextual se hace, como todo en la lengua, con diferentes estructuras del

\footnotetext{
${ }^{12}$ Una propuesta distinta puede consultarse en Garrido (2010, 2011).
} 
sistema: unas más formalizadas, más gramaticalizadas, en forma de palabra o categoría específica, los operadores (modales, informativos, argumentativos, enunciativos). O bien, cuando es necesario una precisión más extensa, o marcas detenidas, se expresa a través de construcciones nominales o clausales.

(17) Señoría, ha hecho usted un batiburrillo, ¿verdad?, un cóctel, una mezcla con diferentes temas que van ustedes cogiendo de los medios de comunicación que, tengo que decirles con absoluta sinceridad, que no sé qué relación guardan con la pregunta que traían ustedes en el día de hoy (DSPA 112, 56, sra. Montero)

Aquí es donde se nos presenta el gran problema. Kaltenböck (2007) habla de no relación, pero entonces ¿cómo solucionar o cómo hablar de oraciones “subordinadas” o coordinadas, en los casos siguientes?

(18) Elena siempre está muy ocupada, lo que es un problema a la hora de concertar una cita

(19) Elena se mostró bastante enfadada- y ella suele ser amable- por lo que el ambiente se enrareció mucho

(20) El trabajo no parece estar muy bien remunerado- $y$ estoy siendo generosa- y el horario es muy malo.

En estos enunciados habría que preguntarse si estamos ante una oración coordinada, y qué coordina. No parece que las dos oraciones estén al mismo nivel. La coordinación parece estar en el nivel o nudo más alto, es decir, afectando al verbo performativo, o acto de habla básico, y coordinando dos actos de decir: "Te digo que Elena se mostró bastante enfadada y que ella suele ser amable”. El segundo actúa como un contraargumento de lo anterior. En (20) sería “Te digo que el trabajo no parece estar muy bien remunerado y que, al decir esto, estoy siendo generosa” Esto último es una información metadiscursiva sobre las implicaciones del acto de habla. Afectaría, pues, al verbo de habla subyacente a todo enunciado ${ }^{13}$.

\footnotetext{
${ }^{13}$ Véase el nudo de inserción sintáctica en la propuesta de Gutiérrez Ordóñez (1997) o en Fuentes Rodríguez (2005, 2007).
} 
6. Las marcas del hablante y su visualización sintáctica en el enunciado

Las propuestas anteriores intentan plantear opciones a la sintaxis del discurso. Todas coinciden en reconocer la necesidad de "etiquetar" las construcciones que apuntan a la inscripción del hablante en su discurso, pero no todas tienen el mismo objetivo. Las comment clauses:

-Reconocen estas funciones modales y enunciativas

-Incluyen la noción semántica de comentario

-Buscan la categorización de una clase de elementos, fijados o semifijados.

El hedging busca categorizar unidades relativas a la enunciación y la modalidad, aunque de forma muy diferente: las que apuntan a la aserción y a la enunciación o decir.

Los estudios sobre parentéticos van más allá. No sólo se abren a funciones como la modalización o enunciación, sino a la expresión de otras etiquetas macroestructurales como la argumentación. Incluyen tanto una clase de elementos fijados, como estructuras sintácticas marginales, hasta enunciados que actúan en este plano. Se estudia, asimismo, el tipo de relación que contraen estas unidades con la oración matriz.

Todo ello, en suma, nos lleva a la necesidad de descripción de la sintaxis del enunciado, que se encuentra con contenidos relacionales y ámbitos diferentes que designar y que pueden sistematizarse en los planos: argumentativo, modal, enunciativo e informativo. Una vez reconocida esta inscripción macroestructural cabe buscar la formalización microestructural sintáctica (Fuentes Rodríguez 2000). Y en esta el primer paso es reconocer que la pausa y la disposición entonativa parentética actúan como una marca formal discriminadora de los elementos que se mueven en estos planos. Por tanto, la marginalidad entonativa constituye una marca de marginalidad sintáctica, en iconicidad perfecta. Los elementos que apuntan a la inscripción del hablante en su discurso contraen una relación de solidaridad, pero no dependencia con la oración. Esta función puede ser cumplida por:

a) Una clase de palabras específica: los operadores. Estos elementos están fijados: provienen de adverbios oracionales o de construcciones sintácticas fijadas 
b) Un tipo de complemento marginal, que actúa en la periferia derecha, izquierda, o intercalado. Puede ser un sintagma o una oración, del tipo de las llamadas "subordinadas adverbiales impropias", pero también una coordinada o una relativa. Estas en muchas ocasiones aportan un contenido diferente a la modalidad, que podemos llamar de comentario, en la que valoran lo expresado en la oración base.

c) Un tipo de enunciados que se insertan, interrumpiendo al enunciado matriz, y alojando indicaciones modales, enunciativas o argumentativas. En estos se incluiría el discurso directo.

Estas unidades, como es evidente, se encuentran en grado diferente de fijación. Hablar de "comment clauses" como una etiqueta para las estructuras de base oracional que aún no se han fijado como operadores puede ser interesante. Aunque, en nuestra opinión, es más rentable utilizar la denominación oraciones de comentario para designar una relación semántico-pragmática que necesita ser etiquetada y que no corresponde, necesariamente, con los segmentos acogidos bajo comment clauses. Por tanto, proponemos que la denominación oraciones o enunciados de comentario queden para ese tipo de relación, y hablemos de operadores modales, enunciativos o argumentativos, o estructuras que van en camino de fijarse como tales (los más avanzados serían los llamados CPR).

La verdadera innovación en esta revisión de propuestas es el reconocimiento del hueco sintáctico ocupado por los complementos o marcas del decir y de la subjetividad, porque esto supone un primer paso en la descripción de la sintaxis del enunciado, e implica reconsiderar o remodelar la todopoderosa y omnipresente sintaxis oracional.

\section{Conclusión}

Como conclusión, podemos afirmar que la sintaxis del enunciado

a) Existe

b) Sigue criterios funcionales

c) Es urgente su formalización, ya que nos serviría para explicar la inserción de muchas de las llamadas subordinadas impropias, pero también a) algunas 
oraciones, entre ellas ciertas relativas, b) las funciones de ciertas coordinadas , y c) por supuesto, ciertas construcciones semifijadas.

d) Es necesario tener en cuenta la posición como característica determinante o funcional en sintaxis. Ya se ha constatado su valor discriminatorio para el adjetivo, diferenciando designación/connotación, pero también contenido proposicional/ coordenadas discursivas. En los complementos verbales y las relaciones oracionales, la posición marca las funciones periféricas: en el margen derecho se incluye la reinterpretación y en el izquierdo los enmarcadores. El paréntesis, como interrupción, se coloca en posición intermedia.

e) Por último, es importante incorporar la diacronía y el estudio de los procesos de gramaticalización para fijar diferentes estructuras según el estadio en el que se encuentren en ese proceso de determinación de las marcas de la subjetividad.

Bibliografía

Alarcos, E. (1973): “Aditamento, adverbio y cuestiones conexas”, en Alarcos, E.: Estudios de gramática funcional del español. Madrid, Gredos, págs. 219-253.

Asher, N. - LAscarides, A. 2003. Logics of Conversation. Cambridge, Cambridge University Press,

ASHER, N. - VIEU, L. (2005): «Subordinating and coordinating discourse relations», Lingua 115, págs. 591-610.

BALLY, C. (1965): Linguistique générale et linguistique française. Berne, Francke, $4^{\circ}$ ed.

Blanche-Benveniste, C. (2002): "Macro-syntaxe et micro-syntaxe: les dispositifs de la rection verbale”, en H.Leth Andersen- H.Nolke (eds), págs. 95-118.

Blanche-Benveniste, C. (2003). Le recouvrement de la syntaxe et de la macrosyntaxe, en A.Scarano (ed.), págs. 53-75.

BRINTON, L.J. (2008): The Comment Clause in English, Cambridge U.P.

BRIZ, A. (1998): El español coloquial en la conversación, Barcelona: Ariel. 
BRIZ, A. - ESTELLÉS, M. (2010): «On the relationship between attenuation, discourse particles and position», en Kaltenböck, G. et al. (eds.), págs. 289-304.

Brown, P. \& Levinson, S. C. (1987 [1978]): Politeness. Some Universal in Language Use. Cambridge, Cambridge University Press.

CAFFI, C. (1999): “On mitigation”, Journal of Pragmatics, 31, págs. 881-909.

CAFFI, C. (2007): Mitigation. Oxford, Elsevier.

CAFFI, C.-R. W.JANNEY (1994): “Toward a pragmatics of emotive communication”, Journal of Pragmatics 22: 325-373.

Dehé, N.- Y. Kavalova (2007): “Parentheticals: an introduction”. En N.DehéY.Kavalova (eds., 2007), págs. 1-22.

Dehé, N.- Y.Kavalova (eds., 2007): Parentheticals. Amsterdam/ Philadelphia, J.Benjamins.

DIK, S.C. (1997): The theory of Functional grammar, 1 y 2, Berlin, Mouton de Gruyter, $2^{a}$ edición revisada. Ed. de K. Hengeveld.

Englebretson, R. (ed. 2007): Stancetaking in discourse, Amsterdam/Philadelphia: J.Benjamins.

EsPinAL, T. (1991): «The representation of disjunct constituents», Language, 67(4), págs. 726-762.

FERNÁNDEZ FERnÁNDEZ, A. (1993): La función incidental en español, Oviedo, Dpto. Filología Española.

Fraser, B. (1975): «Hedged performatives», en Cole, P. y Morgan, J. L. (eds.), Syntax and semantics, Vol. 3, Nueva York, Academic Press, págs. 187-210.

FrASER, B. (2010): «Pragmatic competence: the case of hedging», en Kaltenböck, G. et al. (eds.), págs.15-34.

Fuentes RodRíGuez, C. (1991): “Algunas reflexiones sobre el concepto de modalidad”, RESLA, 7, 93-108. 
FUENTES RODRÍGUEZ, C. (1994): “Significado designativo y significado coordenativo”, Anuario de estudios filológicos 17, págs. 151- 164.

Fuentes RodríGUEZ, C. (1998): “Estructuras parentéticas”, LEA, XX/2, págs. 137-174.

Fuentes RodRíguez, C. (2000): Lingüística pragmática y análisis del discurso, Madrid, Arco Libros.

Fuentes RodríGuez, C. (2003): “Operador/ conector, un criterio para la sintaxis discursiva”, Rilce 19, 1, págs. 61-85.

Fuentes RodríGuez, C. (2005): "Hacia una sintaxis del enunciado”, LEA 27, 1, págs. 33-61.

Fuentes Rodríguez, C. (2007): La sintaxis del enunciado: los complementos periféricos, Madrid, Arco Libros.

FueNTES RodRÍGUEz, C. (2008): “La aproximación enunciativa”, LEA, 30, 2, págs. 223258.

Fuentes RodRíguez, C. (2010): “La aserción parlamentaria: de la modalidad al metadiscurso”, Oralia, 13, págs. 97-125

Fuentes RodríGuez, C. (2012): “El margen derecho del enunciado”, RSEL 42.2, págs. 63-93.

Fuentes Rodríguez, C. (2013): «Las oraciones de comentario en español», en Casanova Herrero, Emili - Calvo Rigual, Cesáreo (eds.): Actes del 26é Congrés de Lingüística i Filologia Romàniques (València, 6-11 de setembre de 2010), Berlin, W. de Gruyter, págs. 3708-3719.

Galán RodríGuez, C. (1999): “La subordinación causal y final”. En Bosque, I. y V. Demonte (dirs.), vol. III, 3597-3642.

GARRIDO, J. (2010): «Thème et dislocation dans la langue parlée: De la syntaxe de la phrase à la construction du discours», Círculo de Lingüística Aplicada a la Comunicación 42: 3-21. http://www.ucm.es/info/circulo/no42/garrido.pdf [21.07.2010] 
GARRIDO, J. (2011): “Unidades y relaciones en la construcción del discurso”, en Actas del IX Congreso de Lingüística General, Valladolid, Universidad de Valladolid: 976-990.

GonZÁLEz RoDRíGUEZ, M. J. (2011): La expresión lingüística de la actitud en el género de opinión: el modelo de la valoración. $R L A$, 49,1, págs. 109-141.

GuTIÉRREZ ORDÓÑEZ, S. (1997): “La determinación de los niveles oracionales”, en La oración y sus funciones. Madrid: Arco Libros, págs. 368-426.

GUTIÉRREZ ORDÓÑEZ, S. (2013): “Sintaxis y pragmática”, conferencia en la Universidad de San Clemente de Ojrid, 2012, en prensa.

HALlidAY, M.A.K. (1985): An Introduction to Functional Grammar, Londres, Edward Arnold.

HALlidAY, Michael A.K. (1994): An introduction to Functional Grammar. Londres: Hodder.

HoBBs, J. R. (1985): “On the Coherence and Structure of Discourse”, Report No. CSLI85-37, Stanford University, Center for the Study of Language and Information.

Hunston, Susan - Geoff Thompson (eds., 2000): Evaluation in Text: Authorial Stance and the Construction of Discourse, Oxford, Oxford University Press.

Kahane,S,-P. Pietrandrea (2011): “Les parenthétiques comme « Unités Illocutoires Associées » Une perspective macrosyntaxique”, en Avanzi, M. \& J. Glikman (éd.), Les Verbes Parenthétiques : Hypotaxe, Parataxe ou Parenthèse ?, Linx, 2011, 61, en prensa.

KALtenBÖCK, G. (2007): “Spoken parenthetical clauses in English. A taxonomy”. En N.Dehé-Y.Kavalova (eds., 2007), págs. 25-52.

KALTENBÖCK, G. et al. (eds.), New approaches to hedging, Bingley, Emerald,

KAPLAN, N. (2004): “Nuevos desarrollos en el estudio de la evaluación en el lenguaje: la teoría de la valoración”, Boletín de Lingüística, 22, págs. 52-78

KARKKAINEN, E. (2003): Epistemic Stance in English Conversation, Amsterdam/Philadelphia: J.Benjamins P.C. 
KEHLER, A. (2002): Coherence, Reference, and the Theory of Grammar, Stanford, CSLI Publications.

KovaccI, O. (1992): El comentario gramatical, Madrid, Arco Libros.

LAKOFF, G. (1973): "Hedges: a study in meaning criteria and the logic of fuzzy concepts”, Journal of Philosophical Logics 2, págs. 458-508.

LAPESA, R. (1975): «Sintaxis histórica del adjetivo calificativo no atributivo», en Homenaje al instituto de filología y literaturas hispánicas Doctor Amado Alonso en su cincuentenario (1923-1973), Buenos Aires, págs. 171-199.

LEMKE, J. L.(1992): "Interpersonal meaning in discourse: Value orientations". En M.Davies- L.Ravelli (eds.): Selected papers in Systemic Linguistics, London, Pinter, págs. 82-104.

LEMKE, J- L. (1998): "Resources for attitudinal meaning: Evaluative orientations in text semantics", Functions of Language 5 (1), págs. 33-56.

LETH, P. (2010): Rhetorical relations and predicate terms, JSM 10: 8th Conference on Semantics and Formal Modelling, LORIA-INRIA, University of Nancy, March 25, 2010. http://jsm.loria.fr/jsm10/documents/abstracts/p53.pdf

Leth Andersen, H.- H. Nolke (eds., 2002): Macro-syntaxe et mcro-sémantique: actes du colloque international d’Arhus, 17-19 mai 2001. Berne, Peter Lang.

LÓPEz SERENA, A. (2012): "En los márgenes de la estructura informativa: la posición inicial de enunciado como 'base’”, LEA, XXXIV, 2, 303-337.

LOUREDA, O.-Acín, E. (eds., 2010): Los estudios sobre marcadores del discurso, hoy. Madrid, Arco Libros.

MackenZie, J.L.-M. Á. Gómez GonzÁLEz (2004): A new architecture for Functional Grammar, Berlin, Mouton de Gruyter.

Mann, W. C. \& Thompson, S. A. (1986): 'Relational Propositions in Discourse', Discourse Processes, vol. 9, no. 1, pp. 57-90.

Martin, J. R. (1995): "Interpersonal meaning, persuasion, and public discourse: Packing semiotic punch", Australian Journal of Linguistics 15: 3-67. 
MARTin, J- R. (2000): “Beyond exchange: Appraisal systems in English”. Evaluation in Text: Authorial Stance and the Construction of Discourse. Eds. Susan HunstonGeoffrey Thompson, Oxford: Oxford University Press, 142-175.

Martin, J. R. (2003): “Introduction. Negotiating heteroglossia: Social perspectives on evaluation”, Text 23, 2, 1-11.

Martin, J. R. (2004): "Mourning: How we get aligned", Discourse and Society 15 (23), págs. 321-344.

Martin, J. R.- P. White (2005): The language of evaluation: Appraisal in English. Hampshire y Nueva York, Palgrave Macmillan.

MARTínEZ, J.A. (1994): “La función incidental y su conexión con otras construcciones del español”, en Cuestiones marginadas de gramática española, Madrid, Istmo, págs. 225-283.

Meyer-Hermann, R. (1988): “Atenuación e intensificación (análisis pragmático de sus formas y funciones en español hablado)”, Anuario de Estudios Filológicos XI, págs. 275-290.

NiemeIER, S. -R. DiRVEN (eds., 1997): The Language of Emotions. Conceptualization. expression, and theoretical foundation, Amsterdam/Philadelphia: John Benjamins P.C.

PALMER, F. R. (1986): Mood and modality, Cambridge, Cambridge U.P.

Peltola, N. (1982/1983): “Comment clauses in Present-Day English. En I. Koskenniemi et al. (eds). Studies in Classical and Modern Philology, págs.101113. Helsinki, Suomalainen Tiedeakatemia.

PolAnYI, L. (1995): 'The Linguistic Structure of Discourse', Technical Report no. CSLI-96-200, Stanford University, Center for the Study of Language and Information.

PrinCE, E. F. et al. (1982): “On hedging in physician-physician discourse, en R. J. Di Pietro (ed.), Linguistics and the Professions. Proceedings of the Second Annual Delaware Symposium on Language Studies, Norwood, Ablex, págs. 83-97. 
QuiRK, R. et al. (1980 [1972]): A Grammar of Contemporary English, London, Longman.

QUIRK, R. et al. (1985): A Comprehensive Grammar of the English Language, London, Longman.

R. A. E. Y Asociación de Academias de la Lengua Española (2009): Nueva Gramática de la lengua española, Madrid, Espasa Libros.

RodRíGuez RAmAlLe, T. (2011): “Sobre si y el margen preverbal en español”, LEA, XXXIII/2, págs. 199-221.

SAntos Río, L. (2003): Diccionario de partículas, Salamanca, Luso-Española de Ediciones.

SCHELFHOUT, C. (2000): “Corpus-based analysis of parenthetical reporting-clauses”. En F. von Eynde et al (eds.): Computational Linguistics in the Netherlands, 1998, Amsterdam: Rodopi, 147-159.

SchneIDER, S, (2007): Reduced parenthetical clauses. A corpus Study of Spoken French, Italian and Spanish, Amsterdam/Philadelphia, Benjamins.

SCHNEIDER, S, (2013): “La atenuación gramatical y léxica”, Oralia, en prensa.

WeINREICH, U. (1966): “On the semantic structure of English”, en J. H. Greenberg (ed.), Universals of Language, Second edition, Cambridge/Mass., MIT Press, págs. 142-217.

White, P. R. (2000): "Dialogue and inter-subjectivity: Reinterpreting the semantics of modality and hedging". En M. Coulthard et al. (eds.): Dialogue analysis VII: Working with dialogue. Selected papers from the 7th international association of dialogue analysis conference. Birmingham/ Tübingen: Neimeyer, págs. 67-80.

White, P. R. (2002): "Appraisal: The language of evaluation and stance". En J.Verschueren et al. (eds.): The handbook of pragmatics. Amsterdam/ Philadelphia: Benjamins, págs. 1-23.

White, P. R. (2003): "Beyond modality and hedging: A dialogic view of the language of intersubjective stance", Text 23 (2), págs. 259-284. 
White, P. R. (2004): "The language of attitude, arguability and interpersonal positioning". Disponible en http://www.grammatics.com/appraisal/index.html. [Consulta: 25/04/2012].

Whichmann, A. (2001): “Spoken parentheticals". En K.Aijmer (ed.): A Wealth of English. Studies in honour of Göran Kjellmer, Göteborg, Acta Universitatis Gothoburgiensis, 177-193.

ZAVADIL, B. (1968): “Medios expresivos de la categoría de modalidad en español”, Iberoamericana Pragensia, 2, 57-86.

Recibido: 9 septiembre 2013

Aceptado: 15 septiembre 2013

Revisado: 30 octubre 3013

Publicado: 31 octubre 2013 